TITLE:

\title{
Environmental Influences on Sleep Behavior in Captive Male Chimpanzees (Pan troglodytes)
}

\section{$\operatorname{AUTHOR}(\mathrm{S}):$}

Morimura, Naruki; Fujisawa, Michiko; Mori, Yusuke; Teramoto, Migaku

\section{CITATION:}

Morimura, Naruki ... [et al]. Environmental Influences on Sleep Behavior in Captive Male Chimpanzees (Pan troglodytes). International Journal of Primatology 2012, 33(4): 822-829

ISSUE DATE:

2012-08

URL:

http://hdl.handle.net/2433/159899

\section{RIGHT:}

The final publication is available at www.springerlink.com; This is not the published version. Please cite only the published version.; この論文 は出版社版でありません。引用の際には出版社版をご確認ご利用くだ さい。 
1) Wildlife Research Center, Kyoto University

2) Chimpanzee Sanctuary Uto 
19

20

\section{Abstract}

Little is known as to the influence of captivity and stressful events on sleep patterns in primates. We investigated the sleep patterns of 19 male chimpanzees living under similar conditions at the Chimpanzee Sanctuary Uto (CSU) in Kumamoto, Japan, using a behavioral sleep index. We conducted nighttime observations of all subjects during a stable period and then observed three subjects after relocation to an unfamiliar facility at CSU. We estimated length of sleep and non-sleep periods over 13-hr video recordings using instantaneous sampling at 1-min intervals to record sleep, which we defined operationally as an inactive posture with the body lying down with the head on the floor or on nesting materials. The 19 subjects slept for a mean \pm SEM of $11.3 \pm 0.26 \mathrm{hr}$ during the stable period, and sleep patterns varied significantly among the subjects. The three relocated subjects all showed temporarily decreased sleep duration in the post-move period but subsequently recovered to the levels observed during the stable period when habituated to the new living quarters. These results suggest that a stressful event may induce temporary sleep shortage lasting for over a week in captive chimpanzees. Sleep patterns may serve as a useful behavioral index of the stress response, as it is less confounded by other behaviors and the actions of human caretakers than other indices.

Keywords: sleep, environmental change, stress, chimpanzee, welfare 


\section{INTRODUCTION}

Sleep quality, measured in humans (Homo sapiens) as the duration and pattern of sleep, has significant effects on physical health and psychological well-being (Anch et al. 1988), but no studies have addressed such effects in non-human primates. However, some studies have investigated intrinsic and extrinsic factors influencing sleep patterns in non-human primates, such as abiotic environmental conditions, social status, and rearing history (Campbell and Tobler 1984). For instance, total sleep time decreases in captive chimpanzees (Pan troglodytes) as maximum relative humidity increases (Videan 2006); the amount of "relaxed" sleep, defined as sleep where the head is held on the chest, is lower in dominant male and female geladas (Theropithecus gelada) compared with lower-ranking group members (Noser et al. 2003), and nursery-reared rhesus macaques (Macaca mulatta) have shorter nocturnal sleep durations than mother-reared monkeys (Barrett et al. 2009). Moreover, total sleep duration correlates negatively with age in geladas (Noser et al. 2003) and humans (Carskadon and Dement 2000), but is positively related to age in owl monkeys (Aotus azarae) (Suzuki and Sri Kantha 2006) and chimpanzees (Videan 2006). Thus, the length of sleep (Carskadon and Dement 2000) could be useful to evaluate the sleep characteristics of primates.

Chimpanzees are a popular primate species for the study of sleep and sleep-related behavior (Anderson 2000). Early studies using both EEG techniques (Adey et al. 1963; McNew et al. 1971) and behavioral observations (Anderson 2000; Riss and Goodall 1976) suggested that lying down in a supine position is a common sleep posture in this 
60

species. Thus, this posture can serve as a simple behavioral index for sleep. Three EEG studies revealed that unrestrained immature chimpanzees slept for 9.7-11.8 hr nocturnally (Bert et al. 1970; Freemon et al. 1971; McNew et al. 1971). In an observational study that defined an awake state as sitting up and engaging in activities such as defecation, urination, or moving about, six captive chimpanzees retired at around 7:05-7:20 p.m. and awoke at around 6:40 a.m. (i.e., 11.2-11.4 hr of nocturnal sleep) (Riss and Goodall 1976). Another study showed that 20 adult captive chimpanzees retired for an average of $10.3 \mathrm{hr}$ and slept for $8.8 \mathrm{hr}$, based on subjects being reclined and remaining immobile for a minimum of 5 min (Videan 2006). Cross-sectional comparison revealed that age and humidity influence the sleep duration of captive chimpanzees (Videan 2006).

Moreover, an observational study reported that the death of a group member also affected the sleep characteristics of group-living captive chimpanzees (Anderson et al. 2010). When one female of the group died, the remaining individuals delayed nesting and changed posture more frequently during the night.

We examined the influence of individual identity, age, and a move to unfamiliar housing (a stressful event) on sleep patterns in captive chimpanzees. First, we investigated 19 captive male chimpanzees to compare individual sleep patterns and investigate the effect of age. Second, we observed the sleep behavior of 3 chimpanzees after they were moved to an unfamiliar facility at CSU to elucidate whether this presumably stressful event affected behavioral sleep patterns. 
81

82

\section{METHODS}

Study Subjects and Sites

We studied chimpanzees housed at the Chimpanzee Sanctuary Uto (CSU; a former laboratory facility) in Kumamoto, Japan (Morimura et al. 2010). All subjects (N=19) were males aged 12-37 years at the beginning of the study. We studied all 19 chimpanzees during a stable period (October 2007-April 2008) when all individuals lived in three independent, all-male groups. The outdoor compounds of these three groups were $128 \mathrm{~m}^{2}\left(\mathrm{~W}: \mathrm{D}: \mathrm{H}=10.0: 12.8: 3.8 \mathrm{~m}\right.$, one group) or $117 \mathrm{~m}^{2}(\mathrm{~W}: \mathrm{D}: \mathrm{H}=8.5: 12.8: 3.8 \mathrm{~m}$, the other two groups) in area. The compounds were surrounded by iron mesh fences and contained vegetation, climbing structures made of logs, fire hoses, hammocks made of burlap sacks, and several feeding devices, for environmental enrichment. Indoor rooms were small chambers of $4 \mathrm{~m}^{2}(\mathrm{~W}: \mathrm{D}: \mathrm{H}=2.0: 2.0: 2.7 \mathrm{~m})$. The subjects remained isolated in indoor rooms from evening until the next morning, but were able to communicate with neighboring individuals visually and physically through iron bars. Indoor rooms were bright from 7:00 a.m.-7:00 p.m., giving a light-dark condition (L:D) of 12:12 each day, with daylight reaching interior rooms through windows on the roof. The indoor rooms were temperature controlled to $20-28^{\circ} \mathrm{C}$; outside air blew into the rooms through a gap in the door, so seasonal changes in the outdoor environment may have influenced indoor conditions.

We also studied three of the chimpanzees, aged 21-25 yr, during a post-move period (February-March 2009). We isolated one male (Takashi) from other chimpanzees during 
102 the night immediately after the move. The other two males (Kazuya and Kenji) spent the

103 night as a pair. All three males were able to access an outdoor compound with different

104 females during daytime hours in their new housing. Their new outdoor compound and

105 indoor rooms were $150 \mathrm{~m}^{2}(\mathrm{~W}: \mathrm{D}: \mathrm{H}=9.0: 15.6: 6.0 \mathrm{~m})$ and $3.6 \mathrm{~m}^{2}(\mathrm{~W}: \mathrm{D}: \mathrm{H}=2.0: 1.8$ :

$1063.5 \mathrm{~m}$ ) in area, respectively, and were similar to the facility described above.

107 Food and water were available ad libitum in this study. The care and use of the

108 chimpanzees complied with the Guide for the Care and Use of Great Apes of the

109 Chimpanzee Sanctuary Uto. This experiment was approved by the CSU and was

110 conducted in a manner commensurate with the ethical policy of the Wildlife Research

111 Center and the Primate Research Institute, Kyoto University, as well as domestic laws

112 related to the welfare and management of animals.

Data Collection

We recorded nocturnal behavior among chimpanzees kept in their indoor rooms using a digital video camera (DCR-SR300; Sony Corp.) equipped with enhanced low-light recording capability (Night Shot; Sony Corp.). We used an infrared LED illuminator (LIR-CA60; Surveillance Tech) to light the space without the subjects' awareness. We recorded images continuously for $13 \mathrm{hr}$, from 5:00 p.m. until 6:00 a.m. the

120 next morning. We recorded the behavior of each subject once per month (i.e. six times)

121 during the stable period. In the post-move period, we recorded behavior once every two 
in the post-move period.

We used instantaneous sampling at $1 \mathrm{~min}$ intervals through the $13 \mathrm{hr}$ period to record sleep, which we defined operationally as an inactive posture with the body lying down with the head on the floor or on nesting materials. We assumed that if an animal was asleep or awake at two consecutive points, then the animal was in that state for the intervening period. The total sleep duration was assumed to be the sum total of the length of sleep bouts for each observation of $13 \mathrm{hr}$.

\section{Statistical Analysis}

To examine influences on length and continuity of sleep behavior, we calculated four variables: total sleep duration as the total length of sleep bouts for each $13 \mathrm{hr}$ observation period; the maximum length of sleep and non-sleep bouts; and the number of sleep bouts per night for each subject. Day length and external temperatures varied during the study period, so we compared the four dependent variables over time (in months) as well as among subjects using two-way analysis of variance (ANOVA) followed by Bonferroni's post hoc tests using the $\mathrm{R}$ computer program (R Development Core Team, 2007). We also examined the relationship between age and the four dependent variables using Pearson's product-moment correlation. Finally, we compared the four variables between the stable and post-move periods and among moved subjects using a two-way ANOVA with Bonferroni's post hoc test. We considered values of $p<0.05$ as statistically significant. 


\section{RESULTS}

The 19 male chimpanzees slept for a mean $( \pm$ SEM) of $11.3 \pm 0.26 \mathrm{hr}$ during the stable period and showed individual differences in total sleep duration $(F(5,18)=11.28, p$ $<0.01)$. Norihei, Shiro, Kanao, and Mikota slept for shorter durations than the rest of the subjects (Fig 1a).

The mean maximum length of a sleep bout per night was $3.1 \pm 0.17 \mathrm{hr}$., and this differed among the 19 chimpanzees $(F(5,18)=1.83, p=0.03$; Fig. 1b), although there were no significant differences in post hoc pairwise comparisons among the subjects. The maximum length of non-sleep bouts per night $(0.5 \pm 0.07 \mathrm{hr})$ also differed among the 19 individuals $(F(5,18)=6.78, p<0.01)$. Six individuals showed longer maximum non-sleep bouts per night than the other subjects (Fig 1c). The number of sleep bouts per night $(24.4 \pm 0.72)$ differed among the 19 subjects $(F(5,18)=3.86, p<0.01)$ and was higher in nine individuals than in the others (Fig. 1d).

The four dependent variables did not vary significantly between months in the stable period (Table I). We found a significant relationship between age and the number of sleep bouts but not between age and the other three sleep variables (Table II). $<<<$ Tables I \& II around here $>>$

In the post-move period, the three chimpanzees slept for a mean duration of $10.0 \pm$

$0.37 \mathrm{hr}$. The change in housing influenced total sleep duration $(F(2,3)=5.17, p<0.01)$, but not the mean maximum length of a sleep bout per night $(F(2,3)=0.19, p=0.90)$, the 
maximum non-sleep bout length per night (mean \pm SEM: $0.5 \pm 0.07 \mathrm{hr}, F(2,3)=2.37, p=$ $0.09)$, or the number of sleep bouts per night $(F(2,3)=1.55, p=0.22)$. Total sleep duration decreased after changing facilities but then increased over time (Fig. 2), reaching the mean total sleep duration of the stable period on the 11th, 9th, and 21 st days after the move for Kazuya, Kenji, and Takashi, respectively.

The individual males showed no difference in total sleep duration $(F(2,3)=2.27, p=$ $0.12)$, the mean maximum length of a sleep bout $(F(2,3)=1.46, p=0.25)$, or the maximum non-sleep bout length $(F(2,3)=2.27, p=0.12)$. However, the mean number of bouts was different among the three subjects $(F(2,3)=6.63, p<0.01)$. Takashi's sleep was more fragmented than that of the other two subjects (post hoc tests: Takashi-Kazuya, $p<0.01$; Takashi-Kenji, $p=0.04$, Kazuya-Kenji; $p=1.00)$.

\section{DISCUSSION}

Mean sleep duration during the stable period was $11.3 \mathrm{hr}$, ranging from $8.7-12.6 \mathrm{hr}$, similar to the results of previous studies (Bert et al. 1970; Freemon et al. 1971; Videan 2006). Age influenced the number of sleep bouts but not the other patterns of these adult male chimpanzees. A longitudinal comparison of sleep patterns between the stable and the post-move period revealed a temporary sleep shortage in chimpanzees moved from one facility to the other. Total sleep duration in the post-move period was significantly 
186 sleep duration increased gradually during the post-move period and reached a level 187 comparable to that observed during the stable period. Therefore, a stressful event (a change of housing) disturbed the sleep of captive chimpanzees in the present study,

189 supporting the results of a study on the effects of the death of a group member on sleep

190 (Anderson et al. 2010).

191 We also identified individual differences in sleep patterns. In particular, Norihei had

192 the shortest total sleep duration and shortest maximum-length sleep bout per night as well

193 as the longest maximum-length non-sleep bout per night during the stable period. A

194 questionnaire study for caretakers suggests that traumatic experiences can cause 195 behavioral abnormalities in captive chimpanzees similar to those observed in 196 post-traumatic stress disorder and depression in humans (Ferdowsian et al. 2010). Sleep

197 shortages and sleep fragmentation in non-human primates are also similar to disturbances observed in human sleep disorders (Adachi et al. 2003; Lévy and Pépin 2003).

199 Chimpanzees might share negative outcomes (e.g., illness or pathology) with humans as a result of sleep disturbances (Averina et al. 2005; Barraud et al. 2009). More intensive studies using behavioral sleep indices should elucidate the relationship between sleep shortages and poor health outcomes in non-human primates.

Abnormal daytime behaviors such as yawning and scratching are commonly used as a proxy for stress responses (Baker and Aureli 1997; Brent 2001; Chamove 1989). However, given that these behaviors are measured during the day, their use as indices may be confounded by other behaviors and the actions of human caretakers. In contrast, sleep is 
207 less influenced by caretakers. Therefore, sleep patterns may serve as an alternative

208 behavioral index of the stress. Further study is necessary to examine the sleep 209 characteristics of captive non-human primates using large samples that include both sexes.

210 A comparative perspective that includes both health problems in captive animals and sleep

211 disorders in humans will aid our understanding of how sleep and stress are related to health

212 and well-being in primates.

214 Acknowledgments

215 This research was supported financially by the Japan Society for the Promotion of 216 Science (JSPS), a Ministry of Education, Culture, Sports, Science, and Technology

217 (MEXT) of Japan Grant-in-Aid for Scientific Research (no. 23650135), and the 2008 IPS

218 Captive Care Grant to author NM and MEXT-20002001 to TM. The current project is 219 also funded by the HOPE project (21-010, AS-22-023) of the Primate Research Institute of Kyoto University (to author NM). Two referees, the editor-in-Chief, Dr. Peter Verbeek and Mr. Michael Seres, provided helpful criticism to improve an earlier version of the manuscript. We also thank the staff members of the Department of Welfare and Sanctuary Uto, for caring for the chimpanzees. 


\section{References}

Adachi, H., Mikamia, A., Kumano-goa, T., Suganumaa, N., Matsumoto, H., Shigedo, Y., Sugita, Y., \& Takeda, M. (2003). Clinical significance of pulse rate rise during sleep as a screening marker for the assessment of sleep fragmentation in sleep-disordered breathing. Sleep Medicine, 4, 537-542.

Adey, W. R., Kado, R. T., \& Rhodes, J. M. (1963). Sleep: Cortical and subcortical recordings in the chimpanzee. Science, 141, 932-933.

Anch, A. M., Browman, C. P., Mitler, M. M., \& Walsh, J. K. (1988). Sleep: a scientific perspective. Englewood Cliffs, NJ: Prentice Hall, Inc.

Anderson, J. R. (2000). Sleep-related behavioural adaptations in free-ranging anthropoid primates. Sleep Medicine Reviews, 4, 355-373.

Anderson, J. R., Gillies, A., \& Lock, L. C. (2010). Pan thanatology. Current Biology, 20, R349-R351.

Averina, M., Nilssen, O., Brenn, T., Brox, J., Arkhipovsky, V.L., \& Kalinin, A.G. (2005). Social and lifestyle determinants of depression, anxiety, sleeping disorders and self-evaluated quality of life in Russia: A population-based study in Arkhangelsk. Social Psychiatry and Psychiatric Epidemiology, 40, 511-518.

Baker, K. C., \& Aureli, F. (1997). Behavioural indicators of anxiety: An empirical test in chimpanzees. Behaviour, 134, 1031-1050.

Barraud, Q., Lambrecq, V., Forni, C., McGuire, S., Hill, M., Bioulac, B., Balzamo, E., Bezard, E., Tison, F., \& Ghorayeb, I. (2009). Sleep disorders in Parkinson's disease: 
The contribution of the MPTP non-human primate model. Experimental Neurology, $219,574-582$.

Barrett, C. E., Noble, P., Hanson, E., Pine, D. S., Winslow, J. T., \& Nelson, E. E. (2009) Early adverse rearing experiences alter sleep-wake patterns and plasma cortisol levels in juvenile rhesus monkeys. Psychoneuroendocrinology, 34, 1029-1040.

252

Bert, J., Kripke, D. F., \& Rhodes, J. (1970). Electroencephalogram of the mature chimpanzee: Twenty-four hour recordings. Electroencephalography and Clinical Neurophysiology, 28, 368-373.

Brent, L. (2001). Behavior and environmental enrichment of individually housed chimpanzees. In L. Brent (Ed), Care and Management of Captive Chimpanzees (pp. 147-171). San Antonio: American Society of Primatologists.

Campbell, S. S., \& Tobler, I. (1984). Animal sleep: A review of sleep duration across phylogeny. Neuroscience \& Biobehavioral Reviews, 8, 269-300.

Carskadon, M.A., \& Dement, W.C. (2000). Normal human sleep: an overview. In M.H. Kryger, T. Roth, W.C. Dement (Eds), Principles and Practice of Sleep Medicine (pp. 13-23). Philadelphia: W.B. Saunders.

Chamove, A. S. (1989). Environmental enrichment: A review. Animal Technology, 40, $155-178$.

Ferdowsian, H.R., Durham, D.L., Kimwele, C., Kranendonk, G., Otali, E., Akugizibwe, T., Mulcahy, J. B. , Ajarova, L., \& Johnson, C. M. (2011). Signs of Mood and Anxiety Disorders in Chimpanzees. PLoS ONE 6, e19855. 
Freemon, F. R., McNew, J. J., \& Adey, W. R. (1971). Chimpanzee sleep stages. Electroencephalography and Clinical Neurophysiology, 31, 485-489.

Lévy, P., \& Pépin, J. L. (2003). Sleep fragmentation: clinical usefulness of autonomic markers. Sleep Medicine, 4, 489-491.

McNew, J. J., Howe, R. C., \& Adey, W. R. (1971) The sleep cycle and subcortical-cortical EEG relations in the unrestrained chimpanzee. Electroencephalography and Clinical Neurophysiology, 30, 489-503.

Morimura, N., Idani, G., \& Matsuzawa, T. (2010). The first chimpanzee sanctuary in Japan: an attempt to care for the "surplus" of biomedical research. American Journal of Primatology, 73, 226-232.

Noser, R., Gygax, L., \&Tobler, I. (2003). Sleep and social status in captive gelada baboons (Theropithecus gelada). Behavioural Brain Research, 147, 9-15.

R Development Core Team. (2007). R: A language and environment for statistical computing. Vienna, Austria: R Foundation for Statistical Computing. Available

Riss, D., \& Goodall, J. (1976). Sleeping behavior and associations in a group of captive chimpanzees. Folia Primatologica, 25, 1-11.

Suzuki, J. \& Sri Kantha, S. (2006). Quantitation of sleep and spinal curvature in an unusually longevous owl monkey (Aotus azarae). Journal of Medical Primatology, $35,321-330$. 
289 Videan, E. N. (2006). Sleep in captive chimpanzee (Pan troglodytes): The effects of

290 individual and environmental factors on sleep duration and quality. Behavioural

291 Brain Research, 169, 187-192.

292 
293 Table I. Results of ANOVAs testing the influence of time on four measures of sleep over

294 the six months of the stable period for 19 adult male chimpanzees.

\begin{tabular}{lcc}
\hline \multicolumn{1}{c}{ Variables } & $F(5,18)$ & P-value \\
\hline Total sleep duration & 1.13 & 0.35 \\
Maximum duration of sleep bout & 0.28 & 0.93 \\
Maximum duration of awake bout & 1.41 & 0.23 \\
Number of sleep bouts & 0.64 & 0.67 \\
\hline
\end{tabular}

295

296

297

298 
299 Table II. Results of Pearson's product-moment correlation tests comparing four sleep

$300 \quad$ variables and age during the stable period for 19 adult male chimpanzees

\begin{tabular}{lccc}
\hline \multicolumn{1}{c}{ Variables } & $r$ & $t$ & P-value \\
\hline Total sleep duration & 0.14 & 1.49 & 0.14 \\
Maximum duration of sleep bout & -0.14 & -1.49 & 0.14 \\
Maximum duration of awake bout & -0.17 & -1.86 & 0.07 \\
Number of sleep bouts & 0.20 & 2.63 & 0.01 \\
\hline
\end{tabular}

301

302 
303 Figure legends

304 Figure 1: Mean \pm SEM (a) total sleep duration, b) maximum length of sleep bout, c) maximum length of non-sleep bout, and d) number of sleep bouts per night for individual chimpanzees during the stable period. Individuals are shown by age in ascending order, from left to right. The dotted line indicates the overall mean for each variable. Significant differences in post hoc pairwise comparisons are indicated by small letters.

310

311 Figure 2: Mean \pm SEM sleep duration during the stable period and three-week blocks during the post-move period for three chimpanzees. Significant differences in post hoc pairwise comparisons are marked by connecting lines. 


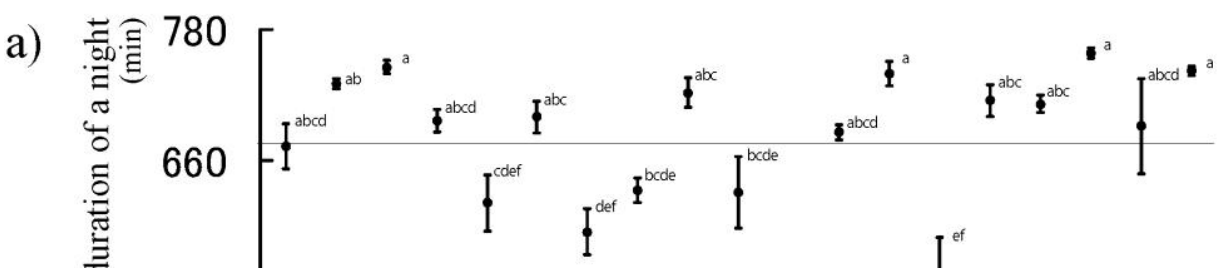

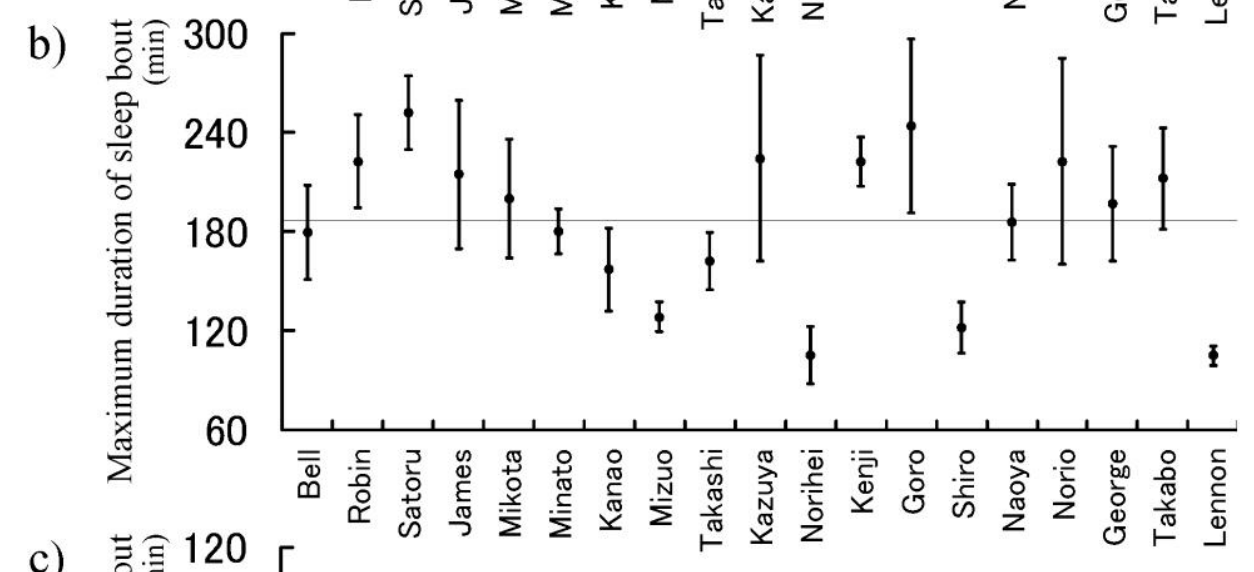

c) 詥 120[

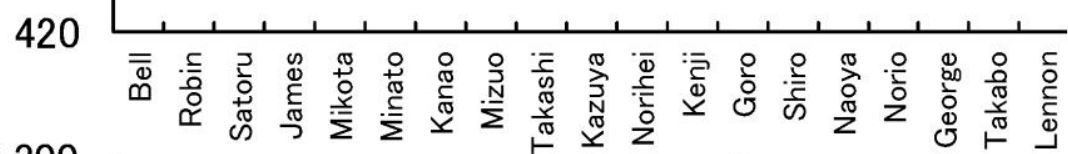

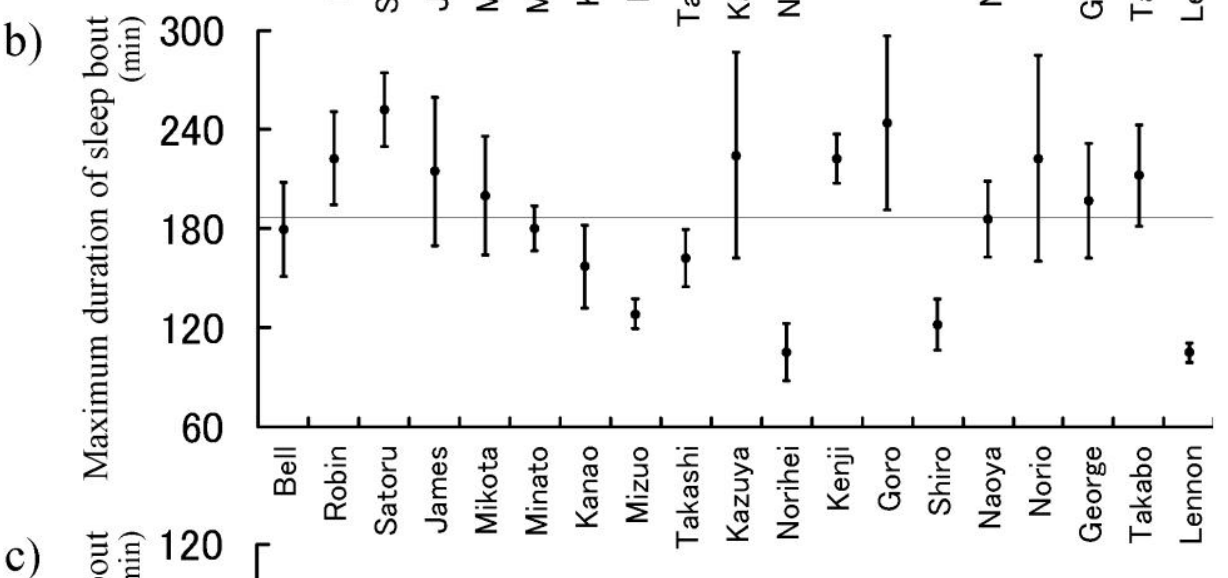

$\ddagger^{\mathrm{f}} \quad \mathfrak{l}^{\mathrm{ef}}$

Figure 1 


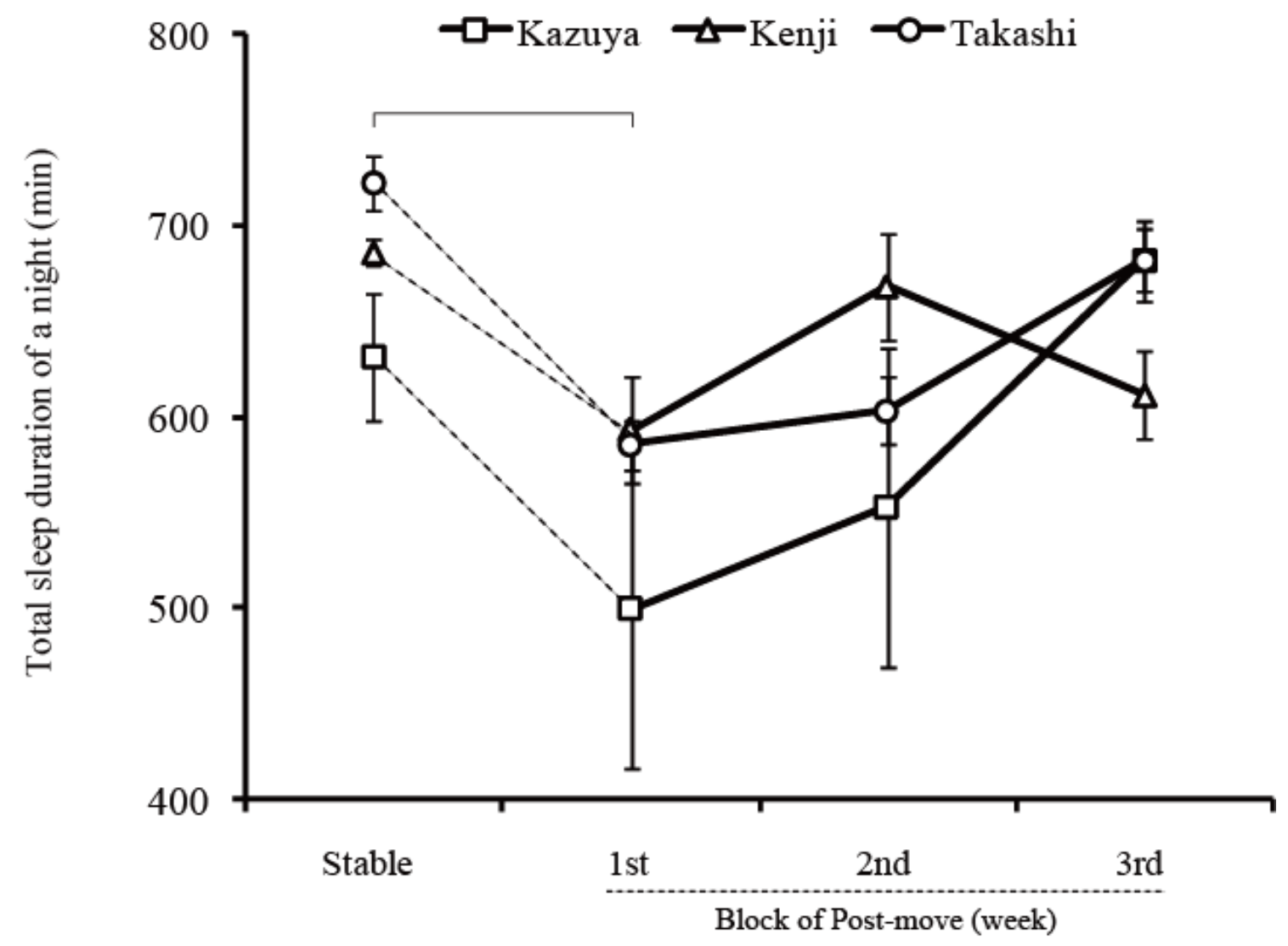

Figure 2 